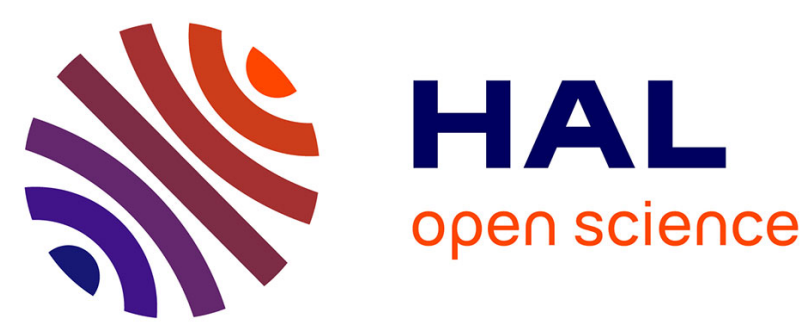

\title{
Kinematic Modeling and Twist Feasibility of Mobile Cable-Driven Parallel Robots
}

\author{
Tahir Rasheed, Philip Long, David Marquez-Gamez, Stéphane Caro
}

\section{To cite this version:}

Tahir Rasheed, Philip Long, David Marquez-Gamez, Stéphane Caro. Kinematic Modeling and Twist Feasibility of Mobile Cable-Driven Parallel Robots. In: Lenarcic J., Parenti-Castelli V. (eds) Advances in Robot Kinematics 2018. ARK 2018. Springer Proceedings in Advanced Robotics, vol 8. Springer, Cham, pp.410-418, 2019, 10.1007/978-3-319-93188-3_47. hal-02405167

\section{HAL Id: hal-02405167 https://hal.science/hal-02405167}

Submitted on 11 Dec 2019

HAL is a multi-disciplinary open access archive for the deposit and dissemination of scientific research documents, whether they are published or not. The documents may come from teaching and research institutions in France or abroad, or from public or private research centers.
L'archive ouverte pluridisciplinaire HAL, est destinée au dépôt et à la diffusion de documents scientifiques de niveau recherche, publiés ou non, émanant des établissements d'enseignement et de recherche français ou étrangers, des laboratoires publics ou privés. 


\title{
Kinematic Modeling and Twist Feasibility of Mobile Cable-Driven Parallel Robots
}

\author{
Tahir Rasheed ${ }^{1}$, Philip Long ${ }^{2}$, David Marquez-Gamez ${ }^{3}$, and Stéphane Caro ${ }^{4}$
}

\begin{abstract}
The kinematic performance of any robotic system can be analyzed from its first order kinematic model. Recently a novel concept of Mobile Cable-Driven Parallel Robots (MCDPRs) has been introduced to achieve an autonomous reconfiguration of Reconfigurable Cable-Driven Parallel Robots (RCDPRs). To analyze the kinematic performance of MCDPRs, this paper presents the first order kinematic model of the latter. Using the proposed kinematic model, the Available Twist Set (ATS) is determined that is required to analyze the twist capacities of MCDPRs. In contrast to ATS of classical CDPRs we show that the ATS of MCDPRs depends, not only on the cables velocities, but on the velocities of Mobile Bases (MBs) as well. As illustrative examples, the twist capacities of two and six Degree of Freedom (DoF) MCDPR is presented.
\end{abstract}

Key words: Cable-Driven Parallel Robots, Mobile Bases, Kinematic Modeling, Available Twist Set

\section{Introduction}

A Cable-Driven Parallel Robot (CDPR) is a type of parallel manipulator with limbs as cables, connecting the moving-platform with a fixed base frame. The platform is moved by appropriately controlling the cable lengths or tensions. CDPRs contains

\footnotetext{
${ }^{1}$ École Centrale de Nantes, Laboratoire des Sciences du Numérique de Nantes, UMR CNRS 6004, 1, rue de la Noë, 44321 Nantes, France, e-mail: Tahir.Rasheed@1s2n.fr, Tahir.Rasheed@irccyn.ecnantes.fr

${ }^{2}$ RIVeR Lab, Department of electrical and computing engineering, Northeastern University, USA e-mail: p.long@northeastern.edu

${ }^{3}$ IRT Jules Verne, Chemin du Chaffault, 44340, Bouguenais, France, e-mail: david.marquezgamez@irt-jules-verne.fr

${ }^{4}$ CNRS, Laboratoire des Sciences du Numérique de Nantes, UMR CNRS 6004, 1, rue de la Noë, 44321 Nantes, France, e-mail: stephane.caro@1s2n.fr
} 
numerous advantages over conventional robots, e.g, high accelerations [6], large payload capabilities [1], and large workspace [7].

However, a major drawback in classical CDPRs having fixed cable layout, i.e, fixed exit points and cable configuration, is the potential collisions between the cables and the surrounding environment which can significantly reduce the robot workspace. Better performances can be achieved with an appropriate CDPR architecture. Cable robots with a possibility of undergoing a change in their geometric structure are known as Reconfigurable Cable-Driven Parallel Robots (RCDPRs). Different strategies have been proposed for maximizing the robot workspace or increasing platform stiffness in the recent work on RCDPRs [3]. However, reconfigurability is typically performed manually for most existing RCDPRs.

To achieve autonomous reconfigurability of RCDPRs, a novel concept of Mobile Cable-Driven Parallel Robots (MCDPRs) was introduced in [10]. The first MCDPR prototype has been designed and built in the context of Echord++ FASTKIT project ${ }^{1}$. The targeted application for such MCDPR prototype is logistics.

Some papers deals with the velocity analysis of parallel manipulators [9]. However, few focus on the twist analysis of CDPRs [8]. This paper deals with the kinematic modeling of MCDPRs that is required to analyze the kinematic performance of the robot. The paper is organized as follows. Section 2 presents the kinematic model of MCDPRs. Section 3 deals with the determination of the Available Twist Set (ATS) for MCDPRs using the kinematic modeling of the latter. The ATS can be used to obtain the twist capacities of the moving-platform. Section 4 presents the twist capacities of the moving-platform for the MCDPRs under study. Finally, conclusions are drawn and future work is presented in Section 5.

\section{Kinematic modeling}

A MCDPR is composed of a classical CDPR with $m$ cables and a $n$ degree-offreedom (DoF) moving-platform mounted on $p$ Mobile Bases (MBs). The $j$ th mobile base is denoted as $\mathscr{M}_{j}, j=1, \ldots, p$. The $i$ th cable mounted onto $\mathscr{M}_{j}$ is named as $\mathscr{C}_{i j}, i=1, \ldots, m_{j}$, where $m_{j}$ denotes the number of cables carried by $\mathscr{M}_{j} . \mathbf{u}_{i j}$ denotes the unit vector along the cable $\mathscr{C}_{i j}$. Each $j$ th mobile base along with its $m_{j}$ number of cables is denoted as $j$ th PD $\left(p d_{j}\right)$ module. Each $p d_{j}$ consists of a proximal $\left(\right.$ prox $\left._{j}\right)$ and a distal $\left(\right.$ dist $\left._{j}\right)$ module. dist $t_{j}$ consists of $m_{j}$ cables between $\mathscr{M}_{j}$ and the moving-platform. In this paper, cables are assumed to be straight and massless, thus can be modeled as a Universal-Prismatic-Spherical (UPS) kinematic chain. Generally MBs are four-wheeled planar robots with two-DoF translational motions and one-DoF rotational motion, thus, prox $_{j}$ can be modeled as a virtual RPP kinematic chain between the base frame $\mathscr{F}_{0}$ and the frame $\mathscr{F}_{b j}$ attached to $\mathscr{M}_{j}$. An illustrative example with $p=4$ MBs and $m=8$ cables is shown in Fig. 1a. A general kinematic architecture of a MCDPR is shown in Fig. 1 .

\footnotetext{
${ }^{1}$ https://www.fastkit-project.eu/
} 

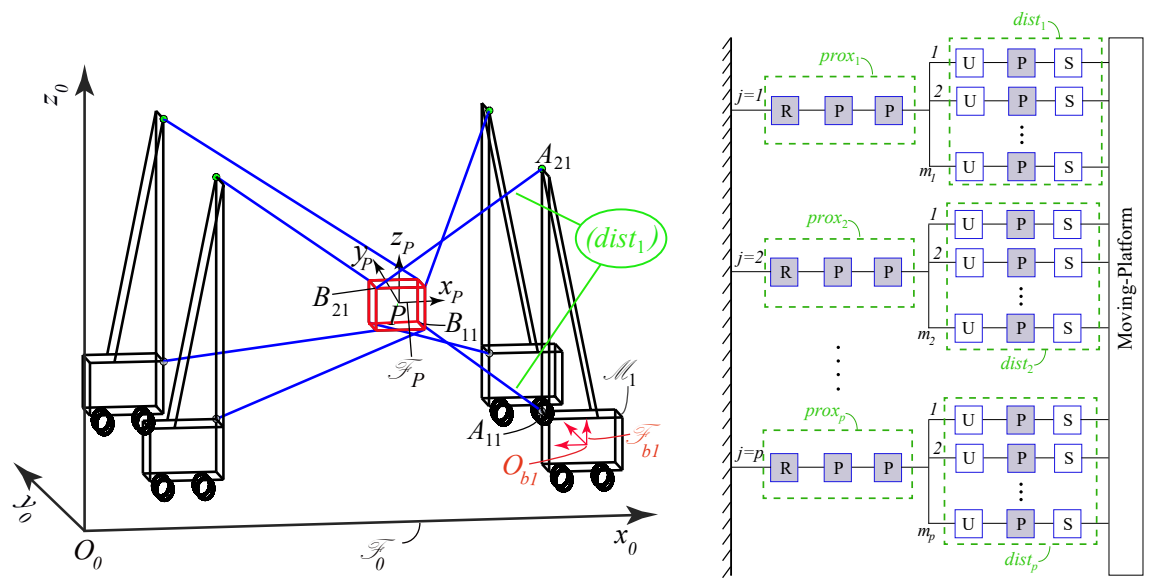

Fig. 1: (a) MCDPR Parameterization (b) Kinematic Architecture of MCDPRs, active joints are highlighted in gray, passive joints are highlighted in white

\subsection{Kinematics of the Distal Module}

A classical CDPR is referred as distal modules in MCDPR. The twist ${ }^{0} \mathbf{t}_{P}^{\text {dist }}$ of the moving-platform due to the latter is expressed as $[4,11]$ :

$$
\mathbf{A}^{0} \mathbf{t}_{P}^{\text {dist }}=\mathbf{i}
$$

where $\mathbf{A}$ is the $(m \times n)$ parallel Jacobian matrix, containing the actuation wrenches due to the cables on the mobile platform. The twist ${ }^{0} \mathbf{t}_{P}=[\boldsymbol{\omega}, \dot{\mathbf{p}}]^{T}$ is composed of the platform angular velocity vector $\boldsymbol{\omega}=\left[\omega_{x}, \omega_{y}, \omega_{z}\right]^{T}$ and linear velocity vector $\dot{\mathbf{p}}=\left[\dot{p}_{x}, \dot{p}_{y}, \dot{p}_{z}\right]^{T}$, expressed in $\mathscr{F}_{0} .{ }^{0} \mathbf{t}_{P}^{\text {dist }}$ denotes the platform twist due to the distal module motion. $\mathbf{i}$ is a $m$-dimensional cable velocity vector. Here, Eq. (1) can be expressed as:

$$
\left[\begin{array}{c}
\mathbf{A}_{1} \\
\mathbf{A}_{2} \\
\vdots \\
\mathbf{A}_{j} \\
\vdots \\
\mathbf{A}_{p}
\end{array}\right]{ }^{0} \mathbf{t}_{P}^{\text {dist }}=\left[\begin{array}{c}
\dot{\mathbf{l}}_{1} \\
\dot{\mathbf{l}}_{2} \\
\vdots \\
\dot{\mathbf{l}}_{j} \\
\vdots \\
\dot{\mathbf{l}}_{p}
\end{array}\right]
$$

where $\mathbf{i}_{j}=\left[\dot{l}_{1 j}, \dot{l}_{2 j}, \ldots, \dot{l}_{m_{j} j}\right]^{T} . \mathbf{A}_{j}$ is expressed as: 


$$
\mathbf{A}_{j}=\left[\begin{array}{cc}
{\left[\left({ }^{0} \mathbf{b}_{1 j}-{ }^{0} \mathbf{p}\right) \times \mathbf{u}_{i j}\right]} & \mathbf{u}_{1 j}^{T} \\
{\left[\left({ }^{0} \mathbf{b}_{2 j}-{ }^{0} \mathbf{p}\right) \times \mathbf{u}_{2 j}\right]^{T}} & \mathbf{u}_{2 j}^{T} \\
\vdots & \\
{\left[\left({ }^{0} \mathbf{b}_{m_{j} j}-{ }^{0} \mathbf{p}\right) \times \mathbf{u}_{m_{j} j}\right]^{T}} & \mathbf{u}_{m_{j} j}^{T}
\end{array}\right],
$$

where $i$ th row of $\mathbf{A}_{j}$ is associated with the actuation wrench of the $i$ th cable mounted onto $\mathscr{M}_{j} .{ }^{0} \mathbf{b}_{i j}$ denotes the Cartesian coordinate vector of the anchor points $B_{i j}$ in $\mathscr{F}_{0}$. ${ }^{0} \mathbf{p}$ denotes the platform position in $\mathscr{F}_{0}$.

\subsection{Kinematic modeling of a MCDPR}

The twist ${ }^{0} \mathbf{t}_{P}^{j}$ of the moving-platform due to $p d_{j}$ can be expressed in $\mathscr{F}_{0}$ as:

$$
{ }^{0} \mathbf{t}_{P}^{j}={ }^{0} \mathbf{t}_{P}^{\text {prox }_{j}}+{ }^{0} \mathbf{t}_{P}^{\text {dist }}
$$

where ${ }^{0} \mathbf{t}_{P}^{\text {prox }_{j}}\left({ }^{0} \mathbf{t}_{P}^{\text {dist }}{ }_{j}\right.$, resp. $)$ is the twist of the moving-platform due to the motion of the proximal (distal, resp.) module of $p d_{j}$ expressed in $\mathscr{F}_{0}$. Equation (4) take the form:

$$
{ }^{0} \mathbf{t}_{P}^{j}={ }^{b j} \mathbf{A} d_{P}{ }^{0} \mathbf{t}_{b j}^{\text {prox }_{j}}+{ }^{0} \overline{\mathbf{R}}_{b j}{ }^{b j} \mathbf{t}_{P}^{\text {dist }}
$$

where ${ }^{b j} \mathbf{A} d_{P}$ is called the adjoint matrix, which represents the transformation matrix between twists expressed in $\mathscr{F}_{b j}$ and twist expressed in $\mathscr{F}_{P}$,

$$
{ }^{b j} \mathbf{A} d_{P}=\left[\begin{array}{cc}
\mathbf{I}_{3} & 0_{3} \\
-{ }^{b j} \hat{\mathbf{r}}_{P} & \mathbf{I}_{3}
\end{array}\right]
$$

where ${ }^{b j} \hat{\mathbf{r}}_{P}$ is the cross-product matrix of vector $\overrightarrow{0_{b j} P}$ expressed in $\mathscr{F}_{0} \cdot{ }^{b j} \mathbf{t}_{P}{ }^{d i s t} t_{j}$ is the moving-platform twist due to dist $j_{j}$ expressed in $\mathscr{F}_{b j}$. The augmented rotation matrix $\left({ }^{0} \overline{\mathbf{R}}_{b j}\right)$ is used to express ${ }^{b j_{i}} \mathbf{t}_{P}{ }^{\text {ist }}{ }_{j}$ in $\mathscr{F}_{0}$ :

$$
{ }^{0} \overline{\mathbf{R}}_{b j}=\left[\begin{array}{cc}
{ }^{0} \mathbf{R}_{b j} & 0_{3} \\
0_{3} & { }^{0} \mathbf{R}_{b j}
\end{array}\right]
$$

where ${ }^{0} \mathbf{R}_{b j}$ is the rotation matrix between frames $\mathscr{F}_{b j}$ and $\mathscr{F}_{0}$. As the Proximal module is being modeled as a virtual RPP limb, ${ }^{0} \mathbf{t}_{b j}{ }^{\text {rrox }_{j}}$ from Eq. (4) can be expressed as:

$$
{ }_{0}^{0} \mathbf{t}_{b j}^{\text {prox }_{j}}=\mathbf{J}_{b j} \dot{\mathbf{q}}_{b j}
$$

where $\mathbf{J}_{b j}$ is a $(6 \times 3)$ serial Jacobian matrix of $\operatorname{prox}_{j}$ and $\dot{\mathbf{q}}_{b j}$ is the virtual joint velocities of the latter, namely, 


$$
{ }^{0} \mathbf{t}_{b j}^{\text {prox }}{ }^{r o}=\left[\begin{array}{ccc}
\mathbf{k}_{0} & \mathbf{0}_{3} & \mathbf{0}_{3} \\
\mathbf{k}_{0} \times{ }^{0} \mathbf{p} & { }^{0} \mathbf{R}_{b j} \mathbf{i}_{0} & { }^{0} \mathbf{R}_{b j} \mathbf{j}_{0}
\end{array}\right]\left[\begin{array}{c}
\dot{\theta}_{j} \\
\dot{\rho}_{1 j} \\
\dot{\rho}_{2 j}
\end{array}\right]
$$

where $\mathbf{i}_{0}, \mathbf{j}_{0}$ and $\mathbf{k}_{0}$ denotes the unit vector along $x_{0}, y_{0}$ and $z_{0}$ respectively. Upon multiplication of Eq. (5) with $\mathbf{A}_{j}$ :

$$
\mathbf{A}_{j}{ }^{0} \mathbf{t}_{P}^{j}=\mathbf{A}_{j}{ }^{b j} \mathbf{A} d_{P} \mathbf{J}_{b j} \dot{\mathbf{q}}_{b j}+\mathbf{A}_{j}{ }^{0} \overline{\mathbf{R}}_{b j}{ }^{b j} \mathbf{t}_{P}^{d i s t_{j}}
$$

As $\mathbf{A}_{j}{ }^{0} \overline{\mathbf{R}}_{b j}{ }^{b j} \mathbf{t}_{P}^{\text {dist }_{j}}$ represents the cable velocities of the dist ${ }_{j}$ (see Eq. (2)), Eq. (10) can also be expressed as:

$$
\mathbf{A}_{j}{ }^{0} \mathbf{t}_{P}^{j}=\mathbf{A}_{j}^{b j} \mathbf{A} d_{P} \mathbf{J}_{b j} \dot{\mathbf{q}}_{b j}+\dot{\mathbf{i}}_{j}
$$

The twist of the moving-platform $\mathbf{t}_{P}$ and the twists generated by the limbs are the same, namely,

$$
{ }^{0} \mathbf{t}_{P}^{1}={ }^{0} \mathbf{t}_{P}^{2}={ }^{0} \mathbf{t}_{P}^{3} \ldots={ }^{0} \mathbf{t}_{P}^{j} \ldots={ }^{0} \mathbf{t}_{P}^{p}=\mathbf{t}_{P}
$$

Thus, the twist of the moving-platform in terms of all the $p$ number of limbs can be expressed as:

$$
\left[\begin{array}{c}
\mathbf{A}_{1} \\
\mathbf{A}_{2} \\
\vdots \\
\mathbf{A}_{p}
\end{array}\right] \mathbf{t}_{P}=\left[\begin{array}{ccccc}
\mathbf{A}_{1}{ }^{b 1} \mathbf{A} d_{P} & \mathbf{J}_{b 1} & \mathbf{0} & \mathbf{0} & \mathbf{0} \\
\mathbf{0} & \mathbf{A}_{2}{ }^{b 2} \mathbf{A} d_{P} & \mathbf{J}_{b 2} & \mathbf{0} & \mathbf{0} \\
\vdots & \vdots & \vdots & \vdots \\
\mathbf{0} & \mathbf{0} & \mathbf{0} \cdots & \mathbf{A}_{p}{ }^{b p} \mathbf{A} d_{P} & \mathbf{J}_{b p}
\end{array}\right] \dot{\mathbf{q}}_{b}+\mathbf{i}
$$

where $\dot{\mathbf{q}}_{b}=\left[\dot{\mathbf{q}}_{b 1}, \dot{\mathbf{q}}_{b 2}, \ldots, \dot{\mathbf{q}}_{b p}\right]^{T}$ and $\dot{\mathbf{i}}=\left[\dot{\mathbf{l}}_{1}, \dot{\mathbf{i}}_{2}, \ldots, \mathbf{i}_{p}\right]^{T}$. Equation (13) can be expressed in the matrix form as:

$$
\begin{gathered}
\mathbf{A t}_{P}=\mathbf{B}_{b} \dot{\mathbf{q}}_{b}+\dot{\mathbf{I}} \\
\mathbf{A} \mathbf{t}_{P}=\mathbf{B} \dot{\mathbf{q}}
\end{gathered}
$$

where $\mathbf{B}=\left[\begin{array}{ll}\mathbf{B}_{b} & \mathbf{I}_{m}\end{array}\right]$ is a $(m \times(3 p+m))$-matrix while $\dot{\mathbf{q}}=\left[\begin{array}{ll}\dot{\mathbf{q}}_{b} & \mathbf{\mathbf { i }}\end{array}\right]^{T}$ is a $(3 p+m)$ dimensional vector containing all joint velocities. Equation (15) represents the first order kinematic model of MCDPRs.

\section{Available Twist Set of MCDPRs}

This section aims at determining the set of available twists for MCDPRs that can be generated by the platform. For a classical CDPR, the set of twist feasible poses of its moving platform are known as Available Twist Set (ATS). A CDPR posture is called twist-feasible if all the twists within a given set, can be produced at the platform, within given joint velocity limits [8]. 
According to [8], ATS of a CDPR corresponds to a convex polytope that can be represented as the intersection of the half-spaces bounded by its hyperplanes known as Hyperplane-Shifting Method (HSM) [2,5]. Although HSM can be utilized to determine the ATS of MCDPRs, the approach in [8] is not directly applicable due to the difference in the kinematic models of CDPR (Eq. 1) and MCDPR (Eq. 15) as matrix $\mathbf{B} \neq \mathbf{I}$.

The kinematic model of the MCDPRs is used to determine the ATS of the moving-platform. In case $m=n, \mathbf{A}$ is square, Eq. (15) can be expressed as:

$$
\mathbf{t}_{P}=\mathbf{A}^{-1} \mathbf{B} \dot{\mathbf{q}} \Longrightarrow \mathbf{t}_{P}=\mathbf{J} \dot{\mathbf{q}}
$$

where $\mathbf{J}$ is a Jacobian matrix mapping the joint velocities onto the platform twist. The ATS will correspond to a single convex polytope, constructed under the mapping of Jacobian $\mathbf{J}$.

In case $m \neq n$, matrix $\mathbf{A}$ is not square, however there exist in total $C_{m}^{n}(n \times n)$ square sub-matrices of matrix $\mathbf{A}$, denoted by $\mathbf{A}^{k}, k=1, \ldots, C_{m}^{n}$, obtained by removing $m-n$ rows from $\mathbf{A}$. For each sub-matrix we can write:

$$
\hat{\mathbf{t}}_{p}^{k}=\mathbf{A}^{k^{-1}} \mathbf{B}^{k} \Longrightarrow \hat{\mathbf{t}}_{p}^{k}=\mathbf{J}^{k} \dot{\mathbf{q}}, \quad k=\left\{1, \ldots, C_{m}^{n}\right\}
$$

where $\hat{\mathbf{t}}_{p}^{k}$ is the twist generated by the $k$ th sub-matrix $\mathbf{A}^{k}$ out of $C_{m}^{n}(n \times n)$ square sub-matrices of matrix $\mathbf{A}$. $\mathbf{B}^{k}$ is a sub matrix of $\mathbf{B}$ using corresponding rows that are chosen in $\mathbf{A}^{k}$ from $\mathbf{A}$. HSM in $[2,5]$ is directly applicable to compute all the hyperplanes for $C_{m}^{n}$ convex polytopes knowing the minimum and maximum joint velocity limits. Thus, the ATS of MCDPRs is the region bounded by all of the foregoing hyperplanes.

\section{Results}

This section deals with the twist feasibility analysis of two different case studies. From the ATS acquired using the kinematic model of a given MCDPR configuration, we aim to study the difference in the moving platform twist considering fixed and moving MBs.

\subsection{Case study: $p=2, m=4$ and $n=2$ DoF MCDPR}

The first case study is a planar MCDPR with a point mass end-effector shown in Fig. 2a. The MBs have only one degree of freedom along $\mathbf{i}_{0}$. The joint velocity limits are defined as:

$$
-0.8 \mathrm{~m} . \mathrm{s}^{-1} \leq \dot{\rho}_{1 j} \leq 0.8 \mathrm{~m} . \mathrm{s}^{-1},-2 \mathrm{~m} . \mathrm{s}^{-1} \leq \dot{i}_{i j} \leq 2 \mathrm{~m} . \mathrm{s}^{-1}, i=\{1,2\}, j=\{1,2\},
$$



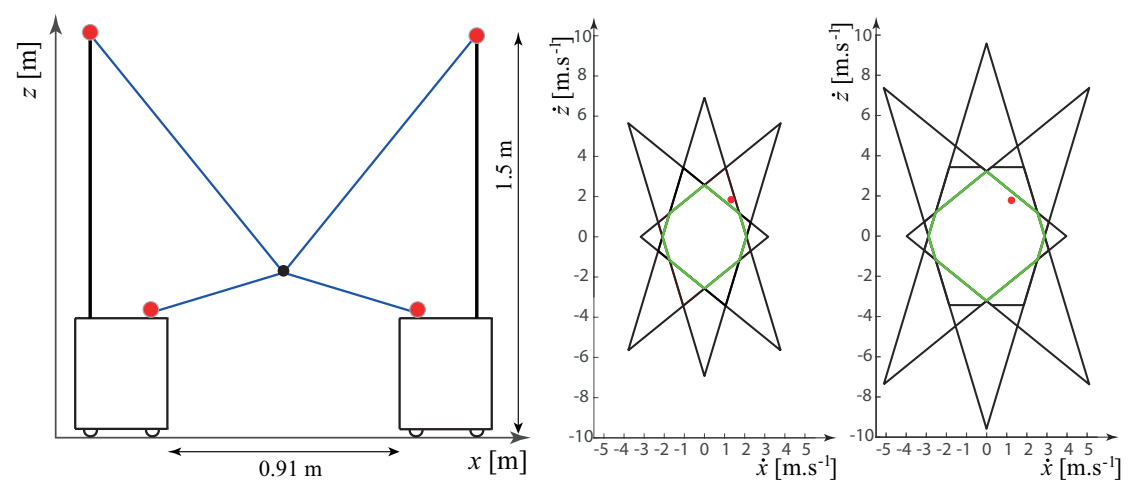

Fig. 2: (a) Configuration under study of $p=2, m=4$ and $n=2$ MCDPR (b) ATS in green for fixed MBs (c) ATS in green for moving MBs

Matrix A has six $2 \times 2$ sub-matrices Thus, ATS is the region bounded by the hyperplanes formed by these six convex polytopes. The difference of ATS between fixed (correspond to a classical CDPR) and moving MBs can be observed in Figs. $2 \mathrm{~b}$ and $2 \mathrm{c}$. To illustrate the difference, a Required Twist Set (RTS) equal to $\left[1.15 \mathrm{~m} . \mathrm{s}^{-1}, 1.675 \mathrm{~m} . \mathrm{s}^{-1}\right]^{T}$ is considered, depicted by a red point in Figs. $2 \mathrm{~b}$ and 2c. For fixed MBs, it should be noted that RTS is outside the ATS. By considering moving MBs, RTS is within the ATS.

\subsection{Case study: $p=2, m=8$ and $n=6$ DoF MCDPR}

The same approach is adopted to determine the ATS for a given FASTKIT configuration in Fig. 3a. The joint velocity limits are defined as:

$$
\begin{aligned}
& -0.2 \mathrm{~m} . \mathrm{s}^{-1} \leq \dot{\theta}_{j}, \dot{\rho}_{1 j}, \dot{\rho}_{2 j} \leq 0.2 \mathrm{~m} . \mathrm{s}^{-1}, \quad j=\{1,2\}, \\
& -2 \mathrm{~m} . \mathrm{s}^{-1} \leq \dot{i}_{i j} \leq 2 \mathrm{~m} . \mathrm{s}^{-1}, \quad j=\{1,2\}, i=\{1, \ldots, 4\},
\end{aligned}
$$

The maximum absolute twist that the platform can achieve in each Cartesian direction by considering fixed and moving MBs are illustrated in Figs. 3b and 3c. The maximum absolute wrench of the moving platform is illustrated in red where $f_{x}, f_{y}, f_{z}$ and $m_{x}, m_{y}, m_{z}$ represent the forces and the moments that can be generated by the cables onto the moving platform. For the analysis, the cable tensions are bounded between 0 and $20 \mathrm{~N}$ respectively. It can be observed the twist capacity of the moving-platform is increased when MBs are moving. On the contrary, high velocity capability of the moving-platform in certain directions also results in very less wrench capability in the respective directions. Thus, this velocity is unattainable outside certain dynamic conditions. 


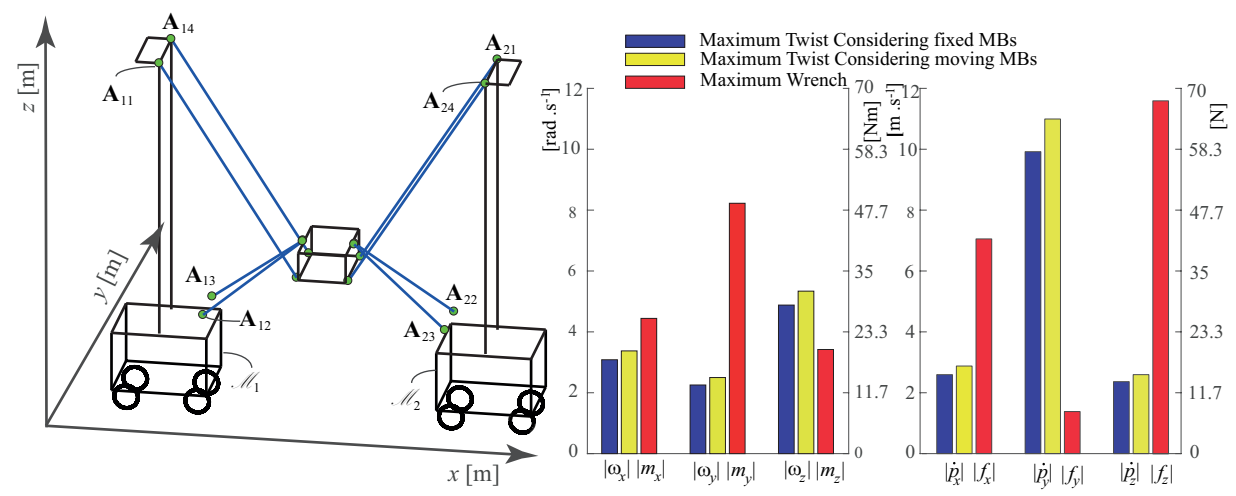

Fig. 3: (a) A FASTKIT configuration (b, c) Maximum absolute twist and wrenches that FASTKIT platform can generate about each Cartesian direction

\section{Conclusion}

This paper dealt with the kinematic modeling of Mobile Cable-Driven Parallel Robots (MCDPRs) that can be used to analyze its kinematic performance. The developed kinematic model was utilized to determine the Available Twist Set (ATS) of MCDPRs. It considers the joint velocity limits for cables and the Mobile Bases (MBs). Using ATS, the twist capacities of the moving-platform was determined. Two case studies have been used in order to illustrate the effect of the moving MBs onto the platform twist. Future work will focus the trajectory planning of of MCDPRs and experimental validations with FASTKIT prototype.

Acknowledgements This research work is part of the European Project ECHORD++ "FASTKIT" dealing with the development of collaborative and mobile cable-driven parallel robots for logistics.

\section{References}

1. J. Albus, R. Bostelman, and N. Dagalakis. The nist robocrane. Journal of Field Robotics, 10(5):709-724, 1993.

2. S. Bouchard, C. Gosselin, and B. Moore. On the ability of a cable-driven robot to generate a prescribed set of wrenches. Journal of Mechanisms and Robotics, 2(1):011010, 2010.

3. L. Gagliardini, S. Caro, M. Gouttefarde, and A. Girin. Discrete reconfiguration planning for cable-driven parallel robots. Mechanism and Machine Theory, 100:313-337, 2016.

4. M. Gouttefarde, D. Daney, and J.-P. Merlet. Interval-analysis-based determination of the wrench-feasible workspace of parallel cable-driven robots. In 2011 IEEE Transactions on Robotics, 27(1):1-13.

5. M. Gouttefarde and S. Krut. Characterization of parallel manipulator available wrench set facets. Advances in robot kinematics: motion in man and machine, pages 475-482, 2010.

6. S. Kawamura, H. Kino, and C. Won. High-speed manipulation by using parallel wire-driven robots. Robotica, 18(1):13-21, 2000. 
7. C. Lambert, M. Nahon, and D. Chalmers. Implementation of an aerostat positioning system with cable control. IEEE/ASME Transactions on Mechatronics, 12(1):32-40, 2007.

8. S. Lessanibahri, M. Gouttefarde, S. Caro, and P. Cardou. Twist feasibility analysis of cabledriven parallel robots. In Cable-Driven Parallel Robots, pages 128-139. Springer, 2018.

9. J.-P. Merlet. Efficient computation of the extremum of the articular velocities of a parallel manipulator in a translation workspace. In 1998 IEEE International Conference on Robotics and Automation (ICRA), volume 3, pages 1976-1981. IEEE, 1998.

10. T. Rasheed, P. Long, D. Marquez-Gamez, and S. Caro. Tension distribution algorithm for planar mobile cable-driven parallel robots. In The Third International Conference on CableDriven Parallel Robots (CableCon 2017), pages 268-279. Springer, 2018.

11. R. G. Roberts, T. Graham, and T. Lippitt. On the inverse kinematics, statics, and fault tolerance of cable-suspended robots. Journal of Field Robotics, 15(10):581-597, 1998. 